\title{
Effect of Maleic Anhydride Content in Properties of PA6/AES Blends Compatibilized with MMA-MA
}

\author{
Amanda Dantas de Oliveira ${ }^{a *}$, Lucas Daniel Chiba de Castro ${ }^{b}$, Cesar Augusto Gonçalves Beatrice ${ }^{b}$, \\ Alessandra de Almeida Lucas ${ }^{b}$ Luiz Antonio Pessan ${ }^{b}$ \\ ${ }^{a}$ Technology Development Center, Federal University of Pelotas, Rua Gomes Carneiro, \\ $n^{\circ}$ 1, 96010-610, Pelotas, RS, Brazil \\ ${ }^{b}$ Department of Materials Engineering, Federal University of São Carlos, via Washington Luis, Km 235, \\ 13565-905, São Carlos, SP, Brazil
}

Received: March 09, 2017; Revised: August 10, 2017; Accepted: August 17, 2017

\begin{abstract}
The effect of maleic anhydride (MA) content on morphological, thermal, thermomechanical and mechanical properties of PA6/AES/MMA-MA (66.5/28.5/5 wt $\%)$ system was investigated. In general, the simple incorporation of MMA-MA to PA6/AES system is responsible for enhancements in mechanical performance. PA6/AES/MMA-MA3\% and PA6/AES/MMA5\% exhibited similar morphology and final properties, indicating no MA content effect on the studied system. On the other hand, PA6/AES/ MMA-MA10\% exhibited a quite different morphology and lower mechanical performance compared to the other compatibilized blends. Such unexpected behavior was not attributed to the effective maleic anhydride content in this composition, but to the reduced molar mass resulted from the excess of nonreacted MA monomer during the synthesis procedure.
\end{abstract}

Keywords: Blends, maleic anhydride, compatibilizer, mechanical properties.

\section{Introduction}

Polyamides belong to the attractive class of engineering thermoplastics due its good strength/stiffness allied to chemical and wear resistance. However, they tend to fail in a brittle manner in the presence of a notch under impact loading ${ }^{1,2}$. Incorporation of elastomeric phases is recognized as an effective approach to minimize such drawbacks of polyamide-based systems $^{3,4}$. Among the wide range of potential toughening agents, acrylonitrile-butadiene-styrene (ABS) shows itself as one of the most popular options. Blends based on PA6/ ABS system are of commercial and academic interest due to their good properties balance allied to competitive cost $^{5,6}$. Nonetheless, the propensity for thermo-oxidative degradation of unsaturated polybutadiene (PB) molecules can limit the lifetime of ABS-toughened molded parts in outdoor applications.

As previously reported ${ }^{7,8}$, such limitations can be overcome by employing saturated elastomers instead of PB. In this way, acrylonitrile-ethylene-propylene-diene-styrene (AES) has been considered as an attractive alternative since it is chemically similar to ABS, except that PB is replaced by ethylene-propylene-diene (EPDM) .

Although, as almost every polymeric pair, PA6/AES blend is immiscible and incompatible due to unfavorable molecular interactions, allowing morphological rearrangements during low stress or quiescent conditions. Unfavorable interactions

*e-mail: amandaoliveira82@gmail.com are also responsible for poor interfacial adhesion in solid state resulting in inferior mechanical properties ${ }^{10}$. Thus, for any PA6/AES based system, the development of an effective compatibilization process is mandatory.

Polymers chemically modified with maleic anhydride (MA) are often used in the compatibilization of polyamidebased materials due to the high probability of in-situ reactions between MA and the amine end-groups of $\mathrm{PA}^{11-13}$. In our previous work ${ }^{10}$, we demonstrated the utility of methyl methacrylate-co-maleic anhydride (MMA-MA) in the compatibilization of PA6/ABS blends. This copolymer contains MA units capable of reacting with PA6 end-groups whereas the methyl methacrylate (MMA) has been demonstrated to be miscible with the SAN phase of ABS over a wide range of acrylonitrile content ${ }^{14}$. Analogously reported by Larocca et al. ${ }^{8}$, once AES is chemically and structurally similar to ABS, it is believed that MMA-MA may also be successfully employed in the compatibilization of PA6/AES blends.

However, depending on investigated system, the amount of available functional groups might have influence on morphology and/or interfacial adhesion obtained through insitu reactions. Borggreve et al. ${ }^{15}$ prepared blends using $90 \mathrm{wt} \%$ of nylon 6 and $10 \mathrm{wt} \%$ of ethylene-propylene-diene rubber (EPDM) grafted with maleic anhydride (MA). The effect of MA concentration on morphology and impact behavior was evaluated. It was found that the use of MA as a coupling agent strongly improves the dispersion of EPDM rubber in the matrix and a refined morphology could be obtained. Nevertheless, within the studied range, MA concentration 
seems to have no influence on toughness of nylon-rubber blends. Li et al. ${ }^{16}$ studied the effect of maleic anhydride (MA) content on nylon 11/ethylene-octene copolymer (POE) system. POE was prior maleated with different MA amounts (POE-g-MA) and posteriorly melt-blended with nylon 11. The authors reported that MA presence could remarkably improve the compatibility between nylon and POE elastomers, enhancing the toughness of blends. The compatibilizing effect on impact strength and reduction of ductile-brittle transition temperature (DBTT) became more pronounced with increasing MA graft ratio until $0.56 \%$, which had been considered the optimal content for the studied system. Kudva et al. ${ }^{17}$ investigated blends of nylon 6 and maleated polyethylene (PE-g-MA) over a range of compositions. Impact properties of nylon 6/PE-g-MA/PE ternary blends were strongly influenced by the molecular weight of the nylon 6 and relative amounts of PE-g-MA and PE. In general, increases in molecular weight of nylon 6 and in ratio of maleated to non-maleated polyethylene leads to enhanced room temperature impact strength and lower ductile-brittle transition temperatures.

From the aforementioned literature, it was found that the effect of functional groups content on compatibilization of multicomponent systems is still not well understood due to the high number of complex interactions occurring simultaneously during melt blending process. Since it is very essential to investigate each case considering their singularities, in the present work, MMA-MA copolymers synthetized with different amounts of MA were employed in an attempt to promote the proper compatibilization of PA6/AES based-systems. Thus, the focus of this study is to investigate the effect of maleic anhydride content in morphological, thermal, thermomechanical and mechanical properties of PA6/AES blends compatibilized with MMA-MA.

\section{Experimental}

Polyamide 6 (PA6) commercialized under tradename

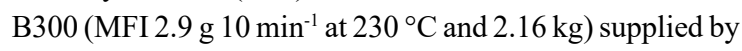
Polyform was employed as the matrix phase. Acrylonitrileethylene-propylene-diene-styrene (AES) commercialized as Royaltuf 372P20 was provided by Chemtura Corporation (50 $\mathrm{wt} \%$ EPDM and $50 \mathrm{wt} \%$ styrene-acrylonitrile) and employed as the toughening agent.

Methyl methacrylate-co-maleic anhydride (MMA-MA) with 3, 5 and $10 \mathrm{wt} \%$ of MA was obtained by solution polymerization. Dimethyl sulfoxide (DMSO) was employed as solvent, azobisisobutyronitrile (AIBN) as reaction initiator, methyl methacrylate (MMA) and maleic anhydride (MA) as monomers; ethyl acrylate (EA) was employed as auxiliary co-monomer to increase the stability of the compatibilizer against unzipping at the processing temperatures ${ }^{18}$. All the monomers were provided by Sigma Aldrich and used without prior purification. The synthesis procedure was performed in a reactor under intensive mixing at $70{ }^{\circ} \mathrm{C}$ and nitrogen atmosphere for $5 \mathrm{~h}$. The obtained product was precipitated in methanol to remove the major part of non-reacted DMSO and MA. Finally, the MMA-MA was filtrated and posteriorly dried in a forced air circulation oven at $50{ }^{\circ} \mathrm{C}$ for $24 \mathrm{~h}^{10,19}$. The effective incorporated MA content was determined by conductometric titration with sodium hydroxide $(\mathrm{NaOH})$ $1 \mathrm{M}$ solution as formerly described by Huang et al. ${ }^{20}$. The synthesis of MMA-MA copolymer is schematically illustrated on Figure 1.

All compositions were prepared by melt blending process using a co-rotational twin screw extruder, B\&P Process Equipment Systems model MP19TC (L/D = 25, $\mathrm{D}=19 \mathrm{~mm}$ ), operating at $230{ }^{\circ} \mathrm{C}$ and $160 \mathrm{rpm}$. MMA-MA synthetized with 3,5 and $10 \mathrm{wt} \%$ of MA were employed to compatibilize PA6/AES blends. Prior to each melt processing step, all polyamide-containing materials were dried in a vacuum oven at $80^{\circ} \mathrm{C}$ for $24 \mathrm{~h}$. The twin screw profile is identical to the one employed on our previous study ${ }^{10}$ and can be observed in Figure 2.

The materials obtained by extrusion were quenched in water, pelletized, dried and injection-molded into standard tensile bars (ASTM D638 type I) and flexural specimens (ASTM D790) using an Arburg Allrounder 270V operating at $245^{\circ} \mathrm{C}$ and mold temperature of $50^{\circ} \mathrm{C}$. The compositions were fixed at (PA6/AES/MMA-MA) (66.5/28.5/5 wt\%). Additionally, a binary (PA6/AES) $(70 / 30 \mathrm{wt} \%$ ) blend was prepared as reference under the same compounding conditions. Samples designation were attributed based on the type of reactive copolymer employed in each composition where the number in front of MMA-MA refers to the nominal fraction of maleic anhydride (MA) employed during the synthesis process.

Molar weights of reactive copolymers were determined by gel permeation chromatography (GPC) on a HP-SEC liquid chromatograph manufactured by Shimadzu equipped with PL Gel 500 columns. Analyzes were carried out at 35 ${ }^{\circ} \mathrm{C}$ using tetrahydrofuran (THF) as solvent.

Differential scanning calorimetry (DSC) analyzes were performed in a DSC Q100 equipment manufactured by TA instruments. Pellets obtained directly from the extrusion process were subjected to heating-cooling-heating cycles from $30{ }^{\circ} \mathrm{C}$ to $250{ }^{\circ} \mathrm{C}$ with constant rate of $10{ }^{\circ} \mathrm{C} \mathrm{min}^{-1}$ under nitrogen atmosphere. Crystallization and melting thermograms were respectively recorded from the first cooling and second heating cycles. Melting $\left(\mathrm{T}_{\mathrm{m}}\right)$ and crystallization temperatures $\left(T_{c}\right)$ were obtained from the DSC curves. The degree of crystallization $\left(\mathrm{X}_{\mathrm{c}}\right)$ were calculated from the second heating cycle as reported elsewhere ${ }^{21}$.

Small angle x-ray scattering (SAXS) measurements were performed in order to study the effect of MA content in the crystalline lamellas of blends. The experiments were conducted at SAX1 beamline of the Brazilian Synchrotron Light Laboratory (LNLS - Brazil). Scatter patterns were 


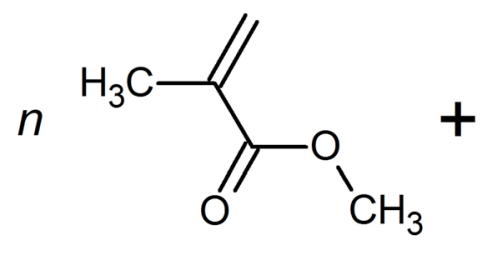

MMA

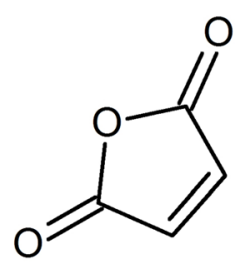

MA

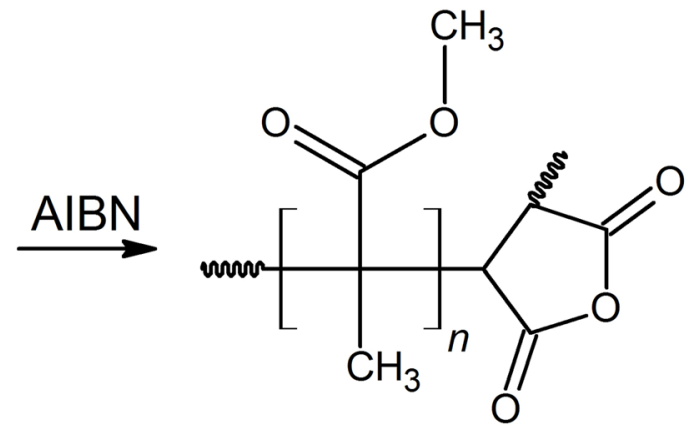

MMA-MA

Figure 1. Synthesis scheme of MMA-MA copolymer.

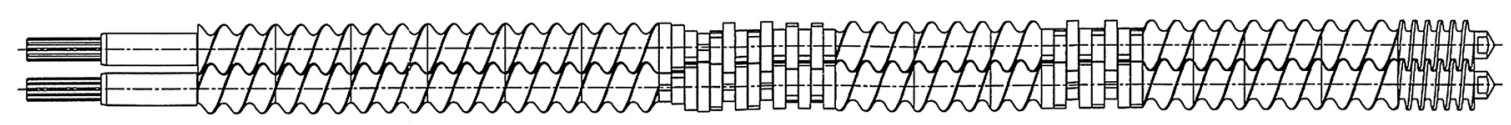

Figure 2. Employed twin screw profile.

collected by a bi-dimensional detector operating with sampleto-detector distance of $459.6510 \mathrm{~mm}$ and $\lambda=1.55 \AA$. Samples were obtained from injection molded specimens. SAXS data were analyzed using the FIT2D software developed by Dr. Andy Hammersley. The long period $\left(\mathrm{L}_{\mathrm{w}}=2 \pi / \mathrm{q}_{\max }\right)$ and crystalline lamellae thickness $\left(\mathrm{L}_{\mathrm{c}}=\mathrm{L}_{\mathrm{w}} \cdot \mathrm{X}_{\mathrm{c}}\right.$, where $\mathrm{X}_{\mathrm{c}}$ is the degree of crystallinity obtained from the DSC scans) of materials were calculated from the Lorentz corrected scattering patterns ${ }^{22}$.

Morphological properties were examined by transmission electron microscopy (TEM) using a Phillips CM120 transmission electron microscope operating at $120 \mathrm{KV}$. The detailed procedure employed to prepare the samples, as well the two-step selective staining technique, is reported elsewhere ${ }^{10}$.

Heat distortion temperature (HDT) was measured according ASTM D 648 (Method A) in a CEAST HDT 6 VICAT P/N 6921.000 equipment operating under outer fiber stress of 1.82 $\mathrm{MPa}$ and heating rate of $120{ }^{\circ} \mathrm{C} \mathrm{h}^{-1}$.

Tensile tests were conducted according to ASTM D638 (type I) using an Instron Model 5568 Universal Testing System. Notched Izod impact tests were performed using a Ceast model Resil 25 pendulum according to ASTM D256-06. All mechanical analyses were carried out at room temperature using dry-as-molded specimens.

\section{Results and Discussions}

\subsection{Characterization of MMA-MA copolymer}

Table 1 summarizes the nominal composition prior to the synthesis procedure and effective incorporated MA fraction in copolymers synthetized with different maleic anhydride (MA) content.
As a general rule, it can be observed that the effective incorporated MA is less than half of the fed amount in the beginning of polymerization process. This phenomenon might be ruled by the considerable reactivity difference between the two monomers $\left(\mathrm{r}_{\mathrm{MMA}}=3.5 \text { and } \mathrm{r}_{\mathrm{MA}}=0.03\right)^{23}$, which clearly is not favorable to the copolymerization process.

From Table 1, subsequent increases in nominal fraction of MA are invariably followed by reductions in both weight average $\left(\mathrm{M}_{\mathrm{w}}\right)$ and number average $\left(\mathrm{M}_{\mathrm{n}}\right)$ molar mass of synthetized copolymers. Due to the poor reactivity of maleic anhydride, it seems that increases MA ratio will not lead to its homopolymerization neither improving the efficiency of grafting reaction, but will just outcome in larger amounts of non-reacted MA monomer dissolved on the system. Furthermore, the low $r_{M A}$ value allied to the decrease of $M_{w}$ and $M_{n}$ with increments in MA nominal fraction for every composition suggest that the non-reacted monomer in excess may act as a terminator of synthesis process, inhibiting the proper growth of copolymer chains.

Still from Table 1, MMA-MA3\% and MMA-MA5\% seem to follow the same pattern and the MA conversion ratio of both copolymers are very close to each other. However, probably the most remarkable result is observed for MMA-MA10\%. Considering the nominal composition, MMA-MA10\% exhibited the lowest maleation efficiency; thus, a considerable amount of non-reacted MA monomer might be expected. Additionally, compared to the other copolymers, MMA-MA10\% also exhibits the very lowest $M_{w}$ and $M_{n}$ values.

According to the afore discussed, it seems reasonable to assume that MMA-MA10\% is mainly constituted by very short MMA-MA copolymer chains from which growth phase was prematurely terminated by the large excess of non-reacted MA monomer. Furthermore, even after the purification process, it 
Table 1. Structural and compositional properties of MMA-MA synthetized with different MA content.

\begin{tabular}{lcccc}
\hline Samples & $\begin{array}{c}\text { Nominal composition } \\
\text { MMA/MA }(\%)\end{array}$ & $\begin{array}{c}\text { Effective incorporated } \\
\text { MA }(\%)\end{array}$ & $\mathrm{M}_{\mathrm{w}}(\mathrm{g} / \mathrm{mol})$ & $\mathrm{M}_{\mathrm{n}}(\mathrm{g} / \mathrm{mol})$ \\
\hline MMA-MA3\% & $97 / 3$ & 1.4 & 47514 & 20674 \\
MMA-MA5\% & $95 / 5$ & 2.1 & 40489 & 16541 \\
MMA-MA10\% & $90 / 10$ & 2.2 & 29018 & 12424 \\
\hline
\end{tabular}

would be wise to regard the possibility that a large portion of residual MA is still present in this composition once, contrary to MMA-MA3\% and MMA-MA5\%, its overwhelming majority did not take part into the grafting reaction. Since fully control grafting reactions is not a trivial task, the impossibility of obtaining reactive copolymers with high incorporated MA content might be related to limitations inherent in the employed synthesis procedure.

\subsection{Thermal properties}

Due to its semi-crystalline nature, structural properties of polyamides might be influenced by any associated chemistry $^{12}$. Maleic anhydride content effect on melting and crystallization behavior of PA6 were evaluated by differential scanning calorimetry (DSC). Thermograms of first cooling and second heating cycles can be respectively observed in Figure 3 (a) and (b). DSC data are summarized in Table 2.
From Figure 3 it is observed the existence of bimodal peaks in second heating thermograms for all compositions. The double melting endotherms is attributed to the polymorphism of PA6, manifested by the coexistence of $\gamma\left(T_{m 2}\right)$ and $\alpha\left(T_{m 3}\right)$ crystalline forms ${ }^{24}$. Still from Figure 3 , one can identify a tinny shoulder around $\mathrm{T}_{\mathrm{m} 1}=192^{\circ} \mathrm{C}$ for PA6 and PA6/AES, which is almost invisible for the compatibilized compositions. From literature ${ }^{25}$, this shoulder is normally related to the formation of less stable crystalline $\alpha$-phase.

According to Table 2, crystallization degree $\left(\mathrm{X}_{\mathrm{c}}\right)$ of PA6 is reduced by AES presence. This behavior was previously reported for PA6/ABS system ${ }^{26}$ and attributed to the retardation of homogenous nucleation and/or inhibition of growth phase during PA6 crystallization process. Due to the aforementioned similarities between both systems, it is believed that such hypothesis can be extended for PA6/AES.
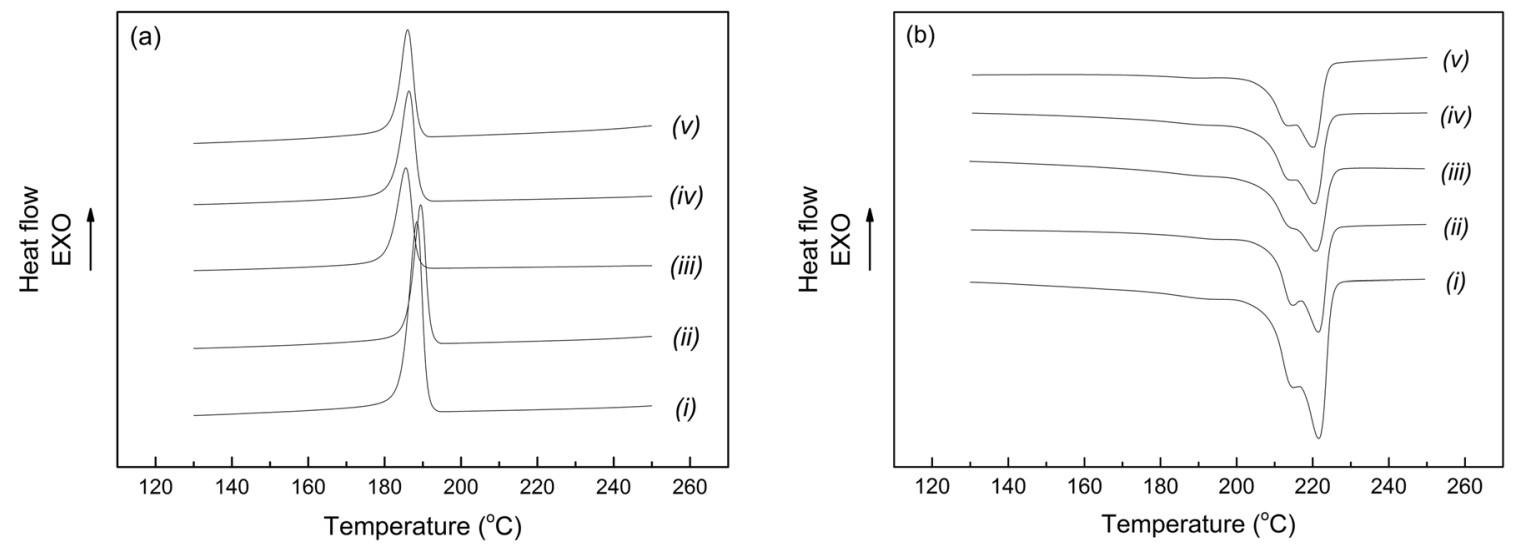

Figure 3. (a) First cooling and (b) second heating thermograms of (i) PA6; (ii) PA6/AES; (iii) PA6/AES/MMA-MA3\%; (iv) PA6/AES/ MMA-MA5\% and (v) PA6/AES/MMA-MA $10 \%$.

Table 2. Summary of DSC characteristic data of neat PA6, PA6/AES and blends compatibilized with MMA-MA synthetized with different MA contents.

\begin{tabular}{lccccccc}
\hline & \multicolumn{3}{c}{ Cooling } & \multicolumn{5}{c}{ Second heating } \\
\cline { 2 - 8 } Samples & $\mathrm{T}_{\mathrm{c}}$ & $\Delta \mathrm{H}_{\mathrm{c}}$ & $\mathrm{T}_{\mathrm{m} 1}$ & $\mathrm{~T}_{\mathrm{m} 2}$ & $\mathrm{~T}_{\mathrm{m} 3}$ & $\Delta \mathrm{H}_{\mathrm{f}}$ & $\mathrm{X}_{\mathrm{c}}$ \\
\cline { 2 - 8 } & $\left({ }^{\circ} \mathrm{C}\right)$ & $\left(\mathrm{J} \mathrm{g}^{-1}\right)$ & $\left({ }^{\circ} \mathrm{C}\right)$ & $\left({ }^{\circ} \mathrm{C}\right)$ & $\left({ }^{\circ} \mathrm{C}\right)$ & $\left(\mathrm{J} \mathrm{g}^{-1}\right)$ & $(\%)$ \\
\hline PA6 & 188 & 65 & 191 & 214 & 222 & 62 & 32 \\
PA6/AES & 188 & 44 & 191 & 214 & 222 & 39 & 29 \\
PA6/AES/MMA-MA3\% & 186 & 39 & - & 214 & 221 & 36 & 28 \\
PA6/AES/MMA-MA5\% & 186 & 40 & - & 213 & 220 & 36 & 28 \\
PA6/AES/MMA-MA10\% & 186 & 35 & - & 213 & 220 & 32 & 25 \\
\hline
\end{tabular}

$T_{\mathrm{c}}:$ crystallization temperature; $\Delta H_{\mathrm{c}}:$ crystallization enthalpy; $T_{\mathrm{m} 1}:$ melting temperature of less stable $\alpha$-phase; $T_{\mathrm{m} 2}:$ melting temperature of $\gamma$-phase; $T_{\mathrm{m} 3}$ : melting temperature of $\alpha$-phase; $\Delta H_{\mathrm{f}}$ : melting enthalpy; $X_{\mathrm{c}}$ : degree of crystallinity 
The presence of MMA-MA slightly reduced both crystallization degree $\left(\mathrm{X}_{\mathrm{c}}\right)$ and crystallization temperatures $\left(\mathrm{T}_{c}\right)$ of ternary blends compared to the binary one. However, despite of such small differences, probably the most noteworthy result is observed for PA6/AES/MMA-MA10\% since it exhibited the lowest $\mathrm{X}_{\mathrm{c}}$ value.

Corroborating the hypothesis raised by GPC data, once the maleation efficiency of synthesis procedure -as well molar masses- of MMA-MA3\% and MMA-MA5\% were approximately the same, the effect of both copolymers in PA6 crystallinity is similar. On the other hand, the reduced $\mathrm{M}_{n}$ and $\mathrm{M}_{\mathrm{w}}$ values of MMA-MA10\% combined to the possible presence of non-reacted MA monomer, might be acting as an additional barrier to the homogeneous nucleation process of PA6, resulting in lower $\mathrm{X}_{\mathrm{c}}$ value.

\subsection{Structural properties}

The Lorentz corrected scattering patterns (I.(q). $\mathrm{q}^{2}$ versus q) obtained for neat PA6, PA6/AES and ternary blends are shown in Figure 4. Assuming that the semicrystalline morphology is formed by isotopically distributed lamellae, i.e. finite crystalline lamellae separated by amorphous layers, Lorentz corrected scattering profiles were used to calculate the long period $\left(\mathrm{L}_{\mathrm{w}}\right)$ and crystalline lamellae thickness $\left(\mathrm{L}_{\mathrm{c}}\right)$. The calculated data are reported in Table 3.

According to Figure 4, a single peak is observed for PA6. Additionally, both binary and ternary blends also exhibited a single peak which are broader than the one observed for PA6. From Table 3 it is verified that such broadening in the peaks is followed by a respective reduction in long period $\left(\mathrm{L}_{\mathrm{w}}\right)$ and crystalline lamellae thickness $\left(\mathrm{L}_{\mathrm{c}}\right)$. It is believed that the decrease in $\mathrm{L}_{\mathrm{c}}$ values allied to the wider pattern might be associated to the formation of thinner and less perfect crystalline lamellae, corroborating the DSC results ${ }^{22}$.

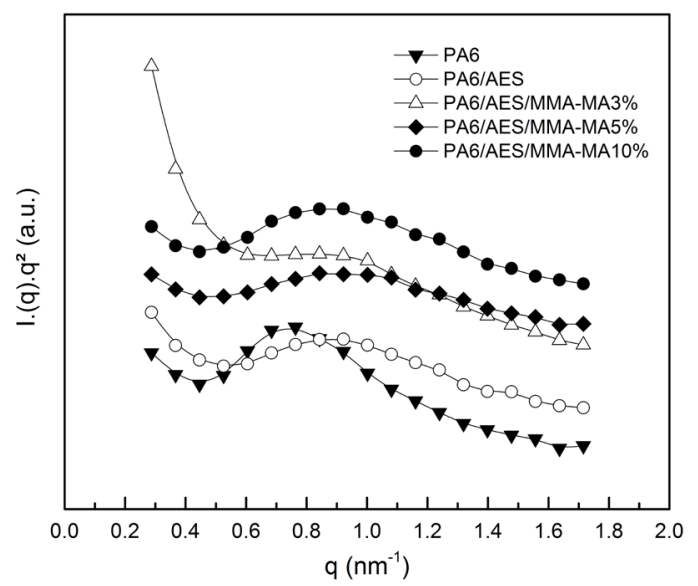

Figure 4. Lorentz corrected SAXS profiles for PA6; PA6/AES and blends compatibilized with MMA-MA synthetized with different MA contents.
Table 3. Calculated data from SAXS measurements of PA6, PA6/ AES and blends compatibilized with MMA-MA synthetized with different MA contents.

\begin{tabular}{lccc}
\hline \multirow{2}{*}{ Samples } & $\mathrm{q}_{\max }$ & $\mathrm{L}_{\mathrm{w}}$ & $\mathrm{L}_{\mathrm{c}}$ \\
\cline { 2 - 4 } & $\left(\mathrm{nm}^{-1}\right)$ & $(\mathrm{nm})$ & $(\mathrm{nm})$ \\
\hline PA6 & 0.74 & 8.49 & 2.72 \\
PA6/AES & 0.88 & 7.13 & 2.07 \\
PA6/AES/MMA-MA3\% & 0.89 & 7.05 & 1.97 \\
PA6/AES/MMA-MA5\% & 0.90 & 6.98 & 1.95 \\
PA6/AES/MMA-MA10\% & 0.89 & 7.05 & 1.76 \\
\hline
\end{tabular}

At this point, an interesting discussion is raised. PA6/ AES/MMA-MA10\% shows $\mathrm{L}_{\mathrm{w}}$ value comparable to the other blends but lower degree of crystallinity. As previously mentioned, it is supposed that the presence of short copolymer chains and/or the possible presence of non-reacted MA monomer on MMA-MA10\%, should hamper the homogenous nucleation process of PA6, reducing its $X_{c}$ value. However, according to SAXS analysis, neither $\mathrm{L}_{\mathrm{w}}$ nor $\mathrm{L}_{\mathrm{c}}$ values seems to be dramatically affected by such singularities. Thus, it is hypothesized that the MMA-MA10\% might affect the homogeneous nucleation of PA6, reducing the number of new crystalline structures and resulting in lower $\mathrm{X}_{\mathrm{c}}$ value; although, once the nucleation process is well-established, the growth of PA6 crystalline phase might not be interfered by MMA-MA10\% molar mass or chemical composition and formation of as thick crystalline lamellae as observed for the other blends is expected.

\subsection{Morphology}

Figure 5 (a-d) show low-magnification TEM micrographs of PA6, PA6/AES and compatibilized blends. The darker part (indicated by black arrows) corresponds to AES while the brighter part corresponds to PA6.

From Figure 5 it is noted that PA6/AES, PA6/AES/ MMA-MA3\% and PA6/AES/MMA-MA5\% morphologies approaches to co-continuous structures and no significant differences between them were observed.

It is undeniable that such morphological similarities are somehow unexpected. Generally speaking, compatibilization processes are normally associated to the reduction of interfacial tension and retardation of coalescence of dispersed phase via steric stabilization, resulting in a finer and more stable structure ${ }^{27-29}$. Nonetheless, it is not necessary to mention that such predictions did not turn into reality for the studied system.

Compared to the other compositions, PA6/AES/MMAMA $10 \%$ exhibit a very unique particle-in-matrix structure, consisted by elongated AES domains barely dispersed in the PA6 matrix. Such anomalous behavior is supposed to be related to the combined effect of small MMA-MA10\% molar mass and low maleation efficiency, which might have hampered the proper dispersion of second phase. 

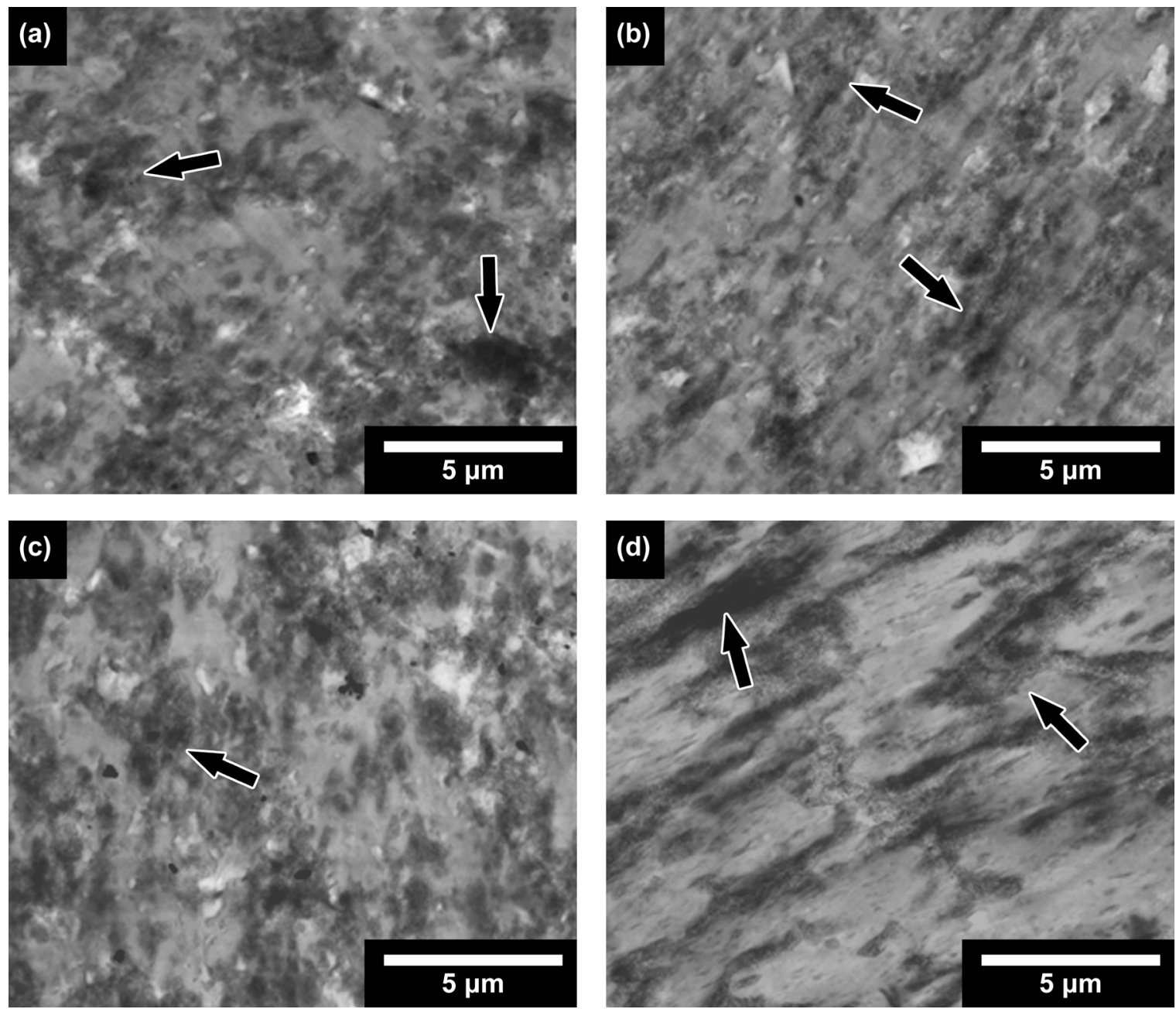

Figure 5. Low-magnification TEM micrographs of (a) PA6/AES; (b) PA6/AES/MMA-MA3\%; (c) PA6/AES/MMA-MA5\% and (d) PA6/ AES/MMA-MA $10 \%$.

Table 4. Thermomechanical and mechanical properties of PA6; PA6/AES and blends compatibilized with MMA-MA synthetized with different MA contents.

\begin{tabular}{lcccc}
\hline Samples & HDT & Izod impact resistance & Modulus & \multicolumn{2}{c}{ Elongation at break } \\
\hline & $\left({ }^{\circ} \mathrm{C}\right)$ & $\left(\mathrm{J} \mathrm{m}^{-1}\right)$ & $(\mathrm{GPa})$ & $35 \pm 7$ \\
\hline PA6 & $53 \pm 1$ & $36 \pm 3$ & $3.2 \pm 0.1$ & $39 \pm 6$ \\
PA6/AES & $60 \pm 2$ & $64 \pm 4$ & $1.8 \pm 0.1$ & $156 \pm 12$ \\
PA6/AES/MMA-MA3\% & $53 \pm 1$ & $223 \pm 10$ & $2.4 \pm 0.1$ & $139 \pm 9$ \\
PA6/AES/MMA-MA5\% & $52 \pm 0$ & $211 \pm 14$ & $2.4 \pm 0.1$ & $50 \pm 14$ \\
PA6/AES/MMA-MA10\% & $52 \pm 1$ & $187 \pm 10$ & & \\
\hline
\end{tabular}

\subsection{Thermomechanical and mechanical properties}

Table 4 summarizes the thermomechanical and mechanical properties of neat PA6, binary and ternary blends. From Table 4 it is possible to observe that AES incorporation dramatically enhanced the heat deflection temperature (HDT) of PA6/AES compared to neat polyamide. The increase in thermal stability due to AES incorporation is attributed to the presence of SAN phase, as reported elsewhere ${ }^{30}$. It is also possible to verify that the incorporation of MMA-MA is followed by a new reduction in HDT values but no MA content effect is observed.

Still from Table 4 one can note that, compared to PA6/ AES, the simple presence of MMA-MA increases the toughness and stiffness of compatibilized systems. At this point, a noteworthy fact should be highlighted. Despite 
the morphological similarities, PA6/AES/MMA-MA3\% and PA6/AES/MMA-MA5\% exhibit improved mechanical properties compared to the non-compatibilized blend.

In a pleasant way, such result came along to reinforce that precociously judging the efficiency of a compatibilizing process by the second phase particle size may be a common, but naïve, mistake. Probably as important as the suppression of coalescence, an effective compatibilizer should provide good adhesion between phases, reducing the possibility of interfacial defects ${ }^{29,31}$.

Among the compatibilized blends, it is observed that PA6/AES/MMA-MA3\% and PA6/AES/MMAMA5\% demonstrated basically the same mechanical performance. Actually, this result sound pretty obvious, since both compositions exhibited lots of similarities along the whole study. As an overall tendency, PA6/AES/ MMA-MA-10\% exhibits lower toughness compared to the other ternary compositions. At this point it seems reasonable to infer that mechanical properties might been hampered by the rough particle-in-matrix structure allied to a poor interfacial adhesion resulted by the low maleation efficiency during the synthesis procedure of MMA-MA10\% allied to it low molar mass.

\section{Conclusions}

In general, MMA-MA incorporation can dramatically enhance the toughness and stiffness of PA6/AES acting as a suitable compatibilizer for this system. PA6/AES/ MMA-MA3\% and PA6/AES/MMA-MA5\% exhibited very similar morphology and mechanical properties, thus, no effect of MA content was observed in the studied system.

On the other hand, PA6/AES/MMA-MA $10 \%$ exhibited some singularities like lower crystallization degree, particle-in-matrix structure and inferior mechanical performance compared to the other compatibilized blends. Such unexpected behavior were attributed to the reduced $M_{n}$ and $M_{w}$ values combined to the possible presence of large amount of non-reacted MA monomer in MMA-MA10\% copolymer. Since the employed synthesis procedure did not show itself as an efficient route for producing MMA-MA with high degree of grafted MA, comparisons involving PA6/AES/MMA-MA10\% data must be careful and, at least for the purpose of this study, should be avoided.

\section{Acknowledgements}

The authors would like to thank Brazilian Counsel of Technological and Scientific Development (CNPq) for the financial support and Brazilian Synchrotron Light Laboratory (LNLS), under proposal D11A-SAXS1-14265, for the SAXS measurements.

\section{References}

1. Kudva RA, Keskkula H, Paul DR. Properties of compatibilized nylon 6/ABS blends: Part I. Effect of ABS type. Polymer. 2000;41(1):225-237.

2. Ozkoc G, Bayram G, Bayramli E. Effects of olefin-based compatibilizers on the morphology, thermal and mechanical properties of ABS/polyamide-6 blends. Journal Applied Polymer Science. 2007;104(2):926-935.

3. Huang JJ, Keskkula H, Paul DR. Rubber toughening of an amorphous polyamide by functionalized SEBS copolymers: morphology and Izod impact behavior. Polymer. 2004;45(12):42034215 .

4. Tjong SC, Xu SA, Mai YW. Impact fracture toughness of short glass fiber-reinforced polyamide 6,6 hybrid composites containing elastomer particles using essential work of fracture concept. Materials Science and Engineering: A. 2003;347(12):338-345.

5. Mojarrad A, Jahani Y, Barikani M. Investigation on the correlation between rheology and morphology of PA6/ABS blends using ethylene acrylate terpolymer as compatibilizer. Journal of Applied Polymer Science. 2011;120(4):2173-2182.

6. Jafari SH, Pötschke P, Stephan M, Warth H, Alberts H. Multicomponent blends based on polyamide 6 and styrenic polymers: morphology and melt rheology. Polymer. 2002;43(25):69856992.

7. Larocca NM, Hage E Jr., Pessan LA. Toughening of poly(butylene terephthalate) by AES terpolymer. Polymer. 2004;45(15):52655277

8. Hage E Jr.,. Effect of reactive compatibilization on the properties of poly(butylene terephthalate)/acrylonitrile-ethylene-propylenediene-styrene blends. Journal of Polymer Science Part B: Polymer Physics. 2005;43(10):1244-1259.

9. Park DJ, Ha CS, Cho WJ. Synthesis and properties of vinyltrimethoxysilane-EPDM-styrene graft terpolymer. Journal of Applied Polymer Science. 1996;67(8):1345-1352

10. Castro LDC, Oliveira AD, Kersch M, Altstädt V, Pessan LA. Effects of mixing protocol on morphology and properties of PA6/ABS blends compatibilized with MMA-MA. Journal of Applied Polymer Science. 2016;133(27).

11. Kudva RA, Keskkula H, Paul DR. Properties of compatibilized nylon 6/ABS blends: Part II. Effects of compatibilizer type and processing history. Polymer. 2000;41(1):239-258.

12. Oshinski AJ, Keskkula H, Paul DR. Rubber toughening of polyamides with functionalized block copolymers: 2 . Nylon-6,6. Polymer. 1992;33(2):284-293.

13. Majumdar B, Keskkula H, Paul DR. Morphology of nylon 6/ABS blends compatibilized by a styrene/maleic anhydride copolymer. Polymer. 1994;35(15):3164-3172.

14. Hale W, Keskkula H, Paul DR. Compatibilization of PBT/ABS blends by methyl methacrylate-glycidyl methacrylate-ethyl acrylate terpolymers. Polymer. 1999;40(2):365-377.

15. Borggreve RJM, Gaymans RJ. Impact behaviour of nylonrubber blends: 4 . Effect of the coupling agent, maleic anhydride. Polymer. 1989;30(1):63-70. 
16. Li QF, Kim DG, Wu DZ, Lu K, Jin RG. Effect of maleic anhydride graft ratio on mechanical properties and morphology on nylon 11/ethylene-octene copolymer blends. Polymer Engineering \& Science. 2001;41(12):2155-2161.

17. Kudva RA, Keskkula H, Paul DR. Morphology and mechanical properties of compatibilized nylon 6/polyethylene blends. Polymer. 1999;40(22):6003-6021.

18. Araújo EM, Hage Jr. E, Carvalho AJF. Morphological, mechanical and rheological properties of nylon 6/acrylonitrile-butadienestyrene blends compatibilized with MMA/MA copolymers. Journal of Materials Science. 2003;38(17):3515-3520.

19. Becker D, Hage Jr. E, Pessan LA. Effects of addition of acrylic compatibilizer on the morphology and mechanical behavior of amorphous polyamide/SAN blends. Journal of Applied Polymer Science. 2010;115(5):2540-2549.

20. Huang Y, Ma X, Liang G, Wang S, Zhang Q. Analysis of the interaction using FTIR within the components of OREC composite GPE based on the synthesized copolymer matrix of P(MMA-MAh). Polymer. 2008;49(8):2085-2094.

21. Kusmono, Ishak ZAM, Chow WS, Takeichi T, Rochmadi. Compatibilizing effect of SEBS-g-MA on the mechanical properties of different types of OMMT filled polyamide 6/ polypropylene nanocomposites. Composites Part A: Applied Science and Manufacturing. 2008;39(12):1802-1814.

22. Bose S, Bhattacharyya AR, Kodgire PV, Misra A, Pötschke P. Rheology, morphology, and crystallization behavior of meltmixed blends of polyamide 6 and acrylonitrile-butadiene-styrene: Influence of reactive compatibilizer premixed with multiwall carbon nanotubes. Journal of Applied Polymer Science. 2007;106(5):3394-3408.
23. Brandrup J, Immergut EH. Polymer Handbook. Hoboken: John Wiley \& Sons; 1965.

24. Liu TX, Liu ZH, Ma KX, Shen L, Zeng KY, He CB. Morphology, thermal and mechanical behavior of polyamide 6/layeredsilicate nanocomposites. Composites Science and Technology. 2003;63(3-4):331-337.

25. Chiu FC, Lai SM, Chen YL, Lee TH. Investigation on the polyamide 6/organoclay nanocomposites with or without a maleated polyolefin elastomer as a toughener. Polymer. 2005;46(25):11600-11609.

26. Bhardwaj IS, Kumar V, Mathur AB, Das A. Characterization of multiphase polymer system nylon 6/ABS blends. Journal of Thermal Analysis. 1990;36(7-8):2339-2347.

27. Kim JK, Kim S, Park CE. Compatibilization mechanism of polymer blends with an in-situ compatibilizer. Polymer. 1997;38(9):2155-2164.

28. Kim BK, Lee YM, Jeong HM. Physical properties of ABS/ SMA/nylon-6 ternary blends: effect of blending sequence. Polymer. 1993;34(10):2075-2080.

29. Sung YT, Han MS, Hyun JC, Kim WN, Lee HS. Rheological properties and interfacial tension of polypropylene-poly(styreneco-acrylonitrile) blend containing compatibilizer. Polymer. 2003;44(5):1681-1687.

30. Oliveira AD, Larocca NM, Paul DR,. Effects of mixing protocol on the performance of nanocomposites based on polyamide 6/ acrylonitrile-butadiene-styrene blends. Polymer Engineering \& Science. 2012;52(9):1909-1919.

31. Díaz MF, Barbosa SE, Capiati NJ. Improvement of mechanical properties for PP/PS blends by in situ compatibilization. Polymer. 2005;46(16):6096-6101. 